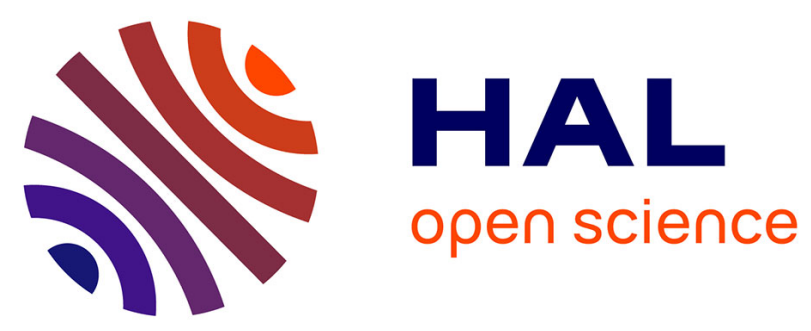

\title{
A comparison of possible disclination mechanisms of switching in thin ferroelectric liquid crystal samples
}

L. Lejček, J.P. Marcerou, C. Destrade, Laurent Dupont

\section{To cite this version:}

L. Lejček, J.P. Marcerou, C. Destrade, Laurent Dupont. A comparison of possible disclination mechanisms of switching in thin ferroelectric liquid crystal samples. Journal de Physique, 1989, 50 (3), pp.361-373. 10.1051/jphys:01989005003036100 . jpa-00210921

\section{HAL Id: jpa-00210921 https://hal.science/jpa-00210921}

Submitted on 1 Jan 1989

HAL is a multi-disciplinary open access archive for the deposit and dissemination of scientific research documents, whether they are published or not. The documents may come from teaching and research institutions in France or abroad, or from public or private research centers.
L'archive ouverte pluridisciplinaire HAL, est destinée au dépôt et à la diffusion de documents scientifiques de niveau recherche, publiés ou non, émanant des établissements d'enseignement et de recherche français ou étrangers, des laboratoires publics ou privés. 


\title{
A comparison of possible disclination mechanisms of switching in thin ferroelectric liquid crystal samples
}

\author{
L. Lejček (*), J. P. Marcerou, C. Destrade and L. Dupont \\ Centre de Recherche Paul Pascal, Domaine Universitaire, 33405 Talence, France
}

(Reçu le 20 mai 1988, révisé le 27 septembre 1988, accepté le 30 septembre 1988)

\begin{abstract}
Résumé. - Trois modèles de nucléation de dislocations : à la surface d'un échantillon ; dans toute son épaisseur ; dans tout son volume ; décrivant le processus de commutation dans un échantillon mince de cristal liquide ferroélectrique (FLC) sont discutés dans l'approximation des couches smectiques parallèles. Les valeurs des champs électriques critiques correspondantes sont estimées. Le processus de commutation entre deux états d'alignement uniforme fait intervenir une combinaison de ces modèles. On discute aussi l'influence d'un ancrage aux surfaces qui peut se modifier pendant la commutation sur la stabilité de l'état d'alignement uniforme obtenu.
\end{abstract}

\begin{abstract}
Three possible disclination models of a nucleation : on a sample surface ; over the whole sample thickness ; in the sample bulk ; describing the switching process in thin ferroelectric liquid crystal (FLC) samples are studied in the approximation of parallel smectic layers and corresponding critical values of electric field are estimated. The switching process between two uniform states of FLC is realized by combination of those models. The influence of a boundary anchoring which can change during switching on the stability of the resulting uniform state is discussed.
\end{abstract}

\section{Introduction.}

Recently, switching processes of a thin surface stabilised ferroelectric liquid crystal cell have been investigated in many studies (see e.g. [1-13]) because thin cells are promising from the point of view of future applications. In such a cell, uniform states with parallel arrangement of molecules can be realized. Molecular anchoring to glass plates is generally planar with contingently inclined smectic layers with respect to the glass surface normal. Layer inclination connected with a dipole-moment inclination from sample surface normal can then cause a « chevron » structure $[7,9-11,13]$ which can result in the occurrence of so called « zigzag " defect $[1,10,12,13]$.

The important mechanism of switching between two uniform states which differ in the molecular dipole-moment orientation is the creation of a nucleus with opposite uniform

(*) On leave from the Institute of Physics, Czechoslovak Academy of Sciences, Na Slovance 2, 18040 Prague 8, Czechoslovakia. 
molecular orientation bounded either by surface or by bulk disclinations [1, 3-8, 11, 13-15].

Recently, elaborated models using a combination of different surface and bulk disclinations were proposed to describe experimental observations $[6,8,11,13-15]$.

In this note we shall study three types of nucleation : (a) on the surface ; (b) over the whole sample thickness, and (c) in the sample bulk, described by surface or bulk disclinations. The switching in thin ferroelectric liquid crystal (FLC) samples under simplifying conditions will be discussed using these nucleation processes and corresponding critical electric fields will be estimated. Our investigation is based on the use of the free energy [14] :

$$
\begin{array}{r}
F=\int_{0}^{h} \mathrm{~d} z \int_{-R}^{R} \mathrm{~d} y \int_{-R}^{R} \mathrm{~d} x\left\{\frac{B_{1}}{2}\left[\left(\frac{\partial \phi}{\partial y}\right)^{2}+\left(\frac{\partial \phi}{\partial z}\right)^{2}\right]+\frac{B_{3}}{2}\left(-\frac{\partial \phi}{\partial x}+q_{0}\right)^{2}-P_{\mathrm{s}} E \cos \phi\right\}+ \\
+\int_{-R}^{R} \mathrm{~d} y \int_{-R}^{R} \mathrm{~d} x\left[\lim _{z \rightarrow 0_{+}} W_{0}(\phi)+\lim _{z \rightarrow h_{-}} W_{h}(\phi)\right] .
\end{array}
$$

In expression (1), $h$ means the sample thickness and $2 R$ its dimensions in the $x$ - and $y$ directions. $B_{1}$ and $B_{3}$ are the FLC elastic constants and $q_{0}$ is connected with the helical pitch $p$ of a perfect FLC structure. $E$ is the z-component of an external electric field and $P_{\mathrm{s}}$ represents the local spontaneous polarization of FLC. We limit our investigation by the following assumptions :

(i) Smectic layers are parallel without «chevron " and «zigzag » defects. The molecular tilt angle in layers is fixed, therefore the molecular orientation will be described by an angle $\phi . \phi$ is the angle between the t-vector which is the projection of molecules onto the smectic layers and the $y$-axis (Fig. 1). The t-vector thus rotates in the $(y z)$ plane.
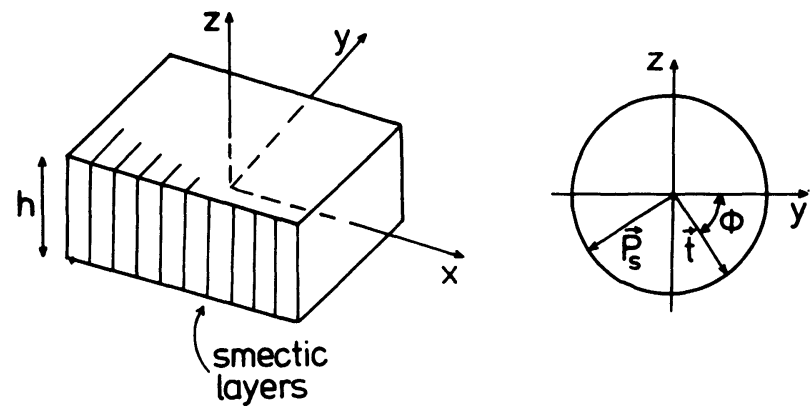

Fig. 1. - Coordinate system in FLC sample. a) The $x$-axis is perpendicular to the smectic layers, the $y$ axis lies along the layers and the $z$-axis is perpendicular to cover glasses. The coordinate origin is situated either on the lower glass plate (see Figs. 2, 3) or in the sample centre (Fig. 4). b) The t-vector which rotates in the $(y, z)$ planes makes an angle $\phi$ with the $y$-axis. The local spontaneous polarisation $\mathbf{P}_{\mathrm{s}}$ is perpendicular to the $\mathrm{t}$-vector.

(ii) There is a planar arrangement of molecules on surfaces, i.e. boundary conditions can be expressed as $\phi=0$ or $\phi= \pm \pi$ at the surfaces.

(iii) The sample is situated between equal glasses, i.e. the upper glass prefers the orientation $\phi=0$ with an anchoring energy $W_{1}$, while the orientation $\phi= \pm \pi$ has the anchoring energy $W_{2}>W_{1}$. The lower glass prefers the orientation $\phi= \pm \pi$ with $W_{1}$ while the anchoring energy $W_{2}$ corresponds to the state $\phi=0$.

(iv) The sample thickness $h$ has such a value that a uniform state is more favorable, i.e. $h<h_{\mathrm{c}}$ where $h_{\mathrm{c}} \approx \pi^{2} B_{1} / 2\left(W_{2}-W_{1}\right)[16]$. 
(v) The external electric field does not modify too much t-vector distribution around disclinations and preferentially stimulates the disclination motion out of the sample which simultaneously leads to the spreading of a new uniform structure over the sample. Using this assumption we can calculate separately the energy of disclination configuration forming the nucleus without an electric field and then we add the approximate energy of the nucleus in an electric field.

According to assumption (iv), the sample is unwound and the contribution to $F$ from terms connected with $q_{0}$ is a constant. For simplicity we put $q_{0}=0$. The values of the surface anchoring energies $W_{0}(\phi)$ and $W_{\mathrm{h}}(\phi)$ in expression (1) at the lower and upper surface, respectively, are defined by assumption (iii).

\section{Nucleus on one sample surface.}

First let us consider the $(-\pi)$ and $(+\pi)$ twist disclinations parallel to the $y$-axis situated on the lower glass plate at $x=-x_{0}$ and $x=x_{0}$ (Fig. 2). The solution which satisfies the equilibrium equation

$$
B_{1} \frac{\partial^{2} \phi}{\partial z^{2}}+B_{3} \frac{\partial^{2} \phi}{\partial x^{2}}=0
$$

for $E=0$ is in the form [14]:

$$
\phi=\operatorname{arctg}\left(\operatorname{th} \frac{\pi\left(x+x_{0}\right)}{2 \alpha h} \operatorname{cotg} \frac{\pi z}{2 h}\right)-\operatorname{arctg}\left(\operatorname{th} \frac{\pi\left(x-x_{0}\right)}{2 \alpha h} \operatorname{cotg} \frac{\pi z}{2 h}\right)
$$

where $\alpha=\left(B_{3} / B_{1}\right)^{1 / 2}$. The solution of (3) fulfils the boundary conditions $\phi(x, z=h)=0$ and

$$
\phi(x, z=0)=\frac{\pi}{2}\left[\operatorname{sg}\left(x+x_{0}\right)-\operatorname{sg}\left(x-x_{0}\right)\right] .
$$

This corresponds to the nucleus of opposite $\mathbf{P}_{\mathrm{s}}$ with respect to the surrounding sample and limited by $( \pm \pi)$-disclination pairs.

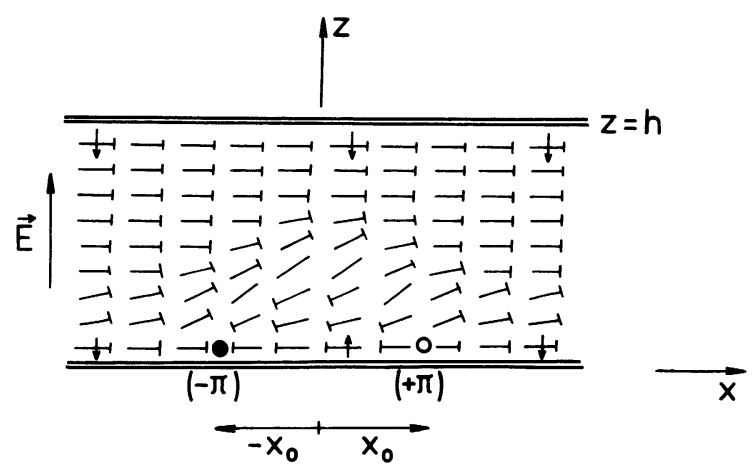

Fig. 2. - Nucleus on the lower sample surface. The molecular arrangement in this and the following figures is schematically represented by nails [17] whose points correspond to the parts of the molecules which are turned toward the observer. Small arrows drawn at some nails represent the orientation of $\mathbf{P}_{\mathrm{s}}$, the direction of the applied electric field $\mathbf{E}$ is shown at the side of drawing. Here (and in the other figures) the projection of $(-\pi)$ disclination into the plane of the figure is represented by a solid circle and the projection of $(+\pi)$ disclination by a circle. Between the dislocations there is the nucleus of molecular structure with $\mathbf{P}_{\mathrm{s}}$ parallel to $\mathbf{E}$. In the sample volume over the nucleus area the molecular twist structure can be distinguished. 
The elastic energy of a nucleus per unit length in the $y$-direction is given by the first integral in (1) with $E=0$ and it is $\pi\left(B_{1} B_{3}\right)^{1 / 2} \ln \left|\operatorname{sh} \frac{\pi x_{0}}{\alpha h}\right| \operatorname{sh} \frac{\pi r_{\mathrm{s}}}{2 \alpha h} \mid$ where $r_{\mathrm{s}}$ is the $\pi$-surface disclination core radius. Within the linear theory based on the free energy (1), the core radius $r_{\mathrm{s}}$ is a parameter. By using the Peierls-Nabarro model $[17,18]$ the core radius can be associated with the maximum surface anchoring energy $W_{\mathrm{M}}$ as $r_{\mathrm{s}} \approx\left(B_{1} B_{3}\right)^{1 / 2} / 2 W_{\mathrm{M}}$.

If surface disclinations are oriented along the $x$-direction the equilibrium equation is then in the form

$$
B_{1}\left(\frac{\partial^{2} \phi}{\partial y^{2}}+\frac{\partial^{2} \phi}{\partial z^{2}}\right)=0
$$

the solution of which has the same form as expression (3) where only $\alpha$ was changed to 1 , and $x$ and $x_{0}$ to $y$ and $y_{0}$, respectively. The parameter $y_{0}$ then describes the positions of $( \pm \pi)$ disclinations parallel to the $x$-axis on the lower surface. The elastic energy of such a nucleus is then $\pi B_{1} \ln \left|\operatorname{sh} \frac{\pi y_{0}}{h} / \operatorname{sh} \frac{\pi r_{\mathrm{s}}}{2 h}\right|$. If the dimensions of the nucleus are $2 x_{0}$ in the $x$ direction and $2 y_{0}$ in the $y$-direction, the elastic energy of such a nucleus can be estimated as

$$
F_{\mathrm{el}}=2 \pi x_{0} B_{1} \ln \left|\operatorname{sh} \frac{\pi y_{0}}{h} / \operatorname{sh} \frac{\pi r_{\mathrm{s}}}{2 h}\right|+2 \pi y_{0}\left(B_{1} B_{3}\right)^{1 / 2} \ln \left|\operatorname{sh} \frac{\pi x_{0}}{\alpha h} / \operatorname{sh} \frac{\pi r_{\mathrm{s}}}{2 \alpha h}\right|,
$$

where the interaction between parallel disclination segments only is taken into account. The surface energy on the upper glass is $4 R^{2} W_{1}$ and on the lower glass is given by

$$
\begin{aligned}
4 x_{0} y_{0} W_{1}+W_{2}\left\{\int_{-R}^{R} \mathrm{~d} x\left[\int_{y_{0}}^{R} \mathrm{~d} y+\int_{-R}^{-y_{0}} \mathrm{~d} y\right]+\int_{-y_{0}}^{y_{0}} \mathrm{~d} y\left[\int_{x_{0}}^{R} \mathrm{~d} x+\int_{-R}^{-x_{0}} \mathrm{~d} x\right]\right\}= \\
=4 R^{2} W_{2}+4 x_{0} y_{0}\left(W_{1}-W_{2}\right)
\end{aligned}
$$

because on the nucleus area $4 x_{0} y_{0}, \phi=-\pi$, with the surface energy $W_{1}$ and outside the nucleus, $\phi=0$ and the surface energy $W_{2}$ (see (iii)).

The energy of an electric field follows from the expression $-\int P_{\mathrm{s}} E \cos \phi \mathrm{d} V$, where $\mathrm{d} V=\mathrm{d} x \mathrm{~d} y \mathrm{~d} z$. Here and in the following parts of this note, assumption $(v)$ will be used. It means that in fact we investigate how the unperturbed structure is favorable when an external electric field is applied. Out of the nucleus $\phi=0$; in the part of the sample over the nucleus: surface $4 x_{0} y_{0}$ we suppose a simple twist, i.e. $\phi=\frac{\pi}{h}(z-h)$. Then

$$
\begin{aligned}
-\int P_{\mathrm{s}} E \cos \phi \mathrm{d} V & =-4 P_{\mathrm{s}} E h\left(R^{2}-x_{0} y_{0}\right)+\int_{-x_{0}}^{x_{0}} \mathrm{~d} x \int_{-y_{0}}^{y_{0}} \mathrm{~d} y \int_{0}^{h} \cos \frac{\pi(z-h)}{h} \mathrm{~d} z \\
& =-4 P_{\mathrm{s}} E h\left(R^{2}-x_{0} y_{0}\right) .
\end{aligned}
$$

The total energy $F$ of a nucleus in our approximation is the sum of expressions (5), (6) and (7) $\ln _{\text {If }}$ the nucleus is rectangular and $x_{0}=y_{0}$ the extremum condition of the total energy with respect to $x_{0}$ is $\partial F / \partial x_{0}=0$ or

$$
\begin{aligned}
& 2 \pi\left(B_{1} B_{3}\right)^{1 / 2}\left\{x_{0} \frac{\pi}{\alpha h}\left[\operatorname{cth} \frac{\pi x_{0}}{\alpha h}+\operatorname{cth} \frac{\pi x_{0}}{h}\right]+\right. \\
& \left.\left[\operatorname{sh} \frac{\pi x_{0}}{\alpha h} / \operatorname{sh} \frac{\pi r_{\mathrm{s}}}{2 \alpha h}\right]+\frac{1}{\alpha} \ln \left|\operatorname{sh} \frac{\pi x_{0}}{h}\right| \operatorname{sh} \frac{\pi r_{\mathrm{s}}}{2 h} \mid\right\}+8 x_{0}\left(W_{1}-W_{2}\right)+8 P_{\mathrm{s}} E h x_{0}=0 .
\end{aligned}
$$


To obtain the orientation $\phi=-\pi$ within the whole sample under the influence of an external electric field we should have $P_{\mathrm{s}} E<0$. When looking for a critical nucleus we can suppose $x_{0}<h$. Then $\operatorname{sh} \frac{\pi x_{0}}{\alpha h} \approx \frac{\pi x_{0}}{\alpha h}, \operatorname{ch} \frac{\pi x_{0}}{\alpha h} \approx 1$ and the equilibrium equation takes the form

$$
2 \pi\left(B_{1} B_{3}\right)^{1 / 2}\left(1+\frac{1}{\alpha}\right) \ln \frac{2 e x_{0}}{r_{\mathrm{s}}}+x_{0}\left(W_{1}-W_{2}\right)=8 x_{0} h\left|P_{\mathrm{s}} E\right|,
$$

where $e \approx 2.71828 \ldots$

Equation (9) gives the dependence of $x_{0}$ on $|E|$. Inserting $x_{0}$ into the expression of the total energy we obtain the total energy of a liquid crystal with a nucleus with molecular polarisation $\mathbf{P}_{\mathrm{s}}$ parallel to $\mathbf{E}$. Comparison of such a total energy with the thermal energy $k T$ where ( $k$ is the Boltzman constant and $T$ the absolute temperature) then gives the temperature dependence of the critical field $E_{\mathrm{c}}^{\mathrm{I}}$ necessary for the nucleation process. However, the upper limit of the critical field can be estimated by considering the smallest possible nucleus, i.e. $x_{0} \approx r_{\mathrm{s}}$. Then

$$
\left|E_{\mathrm{c}}^{\mathrm{I}}\right| \approx \frac{W_{1}-W_{2}}{h\left|P_{\mathrm{s}}\right|}+\frac{\pi\left(B_{1} B_{3}\right)^{1 / 2}}{4 r_{\mathrm{s}} h\left|P_{\mathrm{s}}\right|}\left(1+\frac{1}{\alpha}\right) \ln (2 e)
$$

Because $W_{1}-W_{2}<0$, the term $\frac{W_{1}-W_{2}}{h\left|P_{s}\right|}$ supports the nucleation process by a decrease of $\left|E_{\mathrm{c}}^{\mathrm{I}}\right|$. As in reference [14] it can be shown that conditions (8) and (9) correspond to the maximum energy. When this maximum energy is reached, the nucleus spreads over the whole sample or, at least, over part of it. In this case the nucleation process induces the transition from uniform to twisted structure (Fig. 2).

\section{Nucleus over the whole thickness of a thin FLC sample.}

A generalization of the situation described in section 2 is shown in figure 3 where the nucleation of a second pair of $( \pm \pi)$ surface twist disclinations occurs. First the disclinations are parallel to the $y$-axis. The solution $\phi$ of the equilibrium equation (2) is composed of two terms $\phi_{1}$ and $\phi_{2}$ :

$$
\phi=\phi_{1}+\phi_{2}
$$

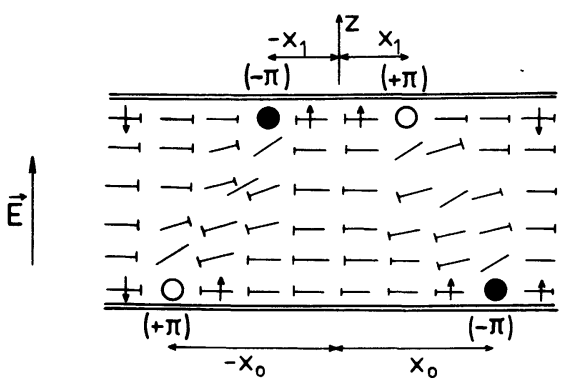

a)

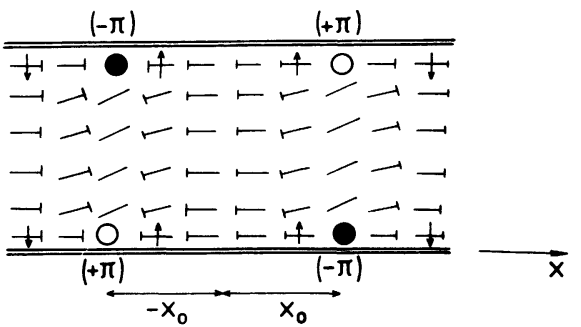

b)

Fig. 3. - Nucleus of the uniform structure over the whole sample thickness. a) The uniform structures with opposite $\mathbf{P}_{\mathrm{s}}$ are separated by twist walls composed of surface $(\mp \pi)$ twist disclination pairs. b) Simplified case when the surface disclinations on opposite glass covers are situated above each other. 
where

$$
\phi_{1}=\operatorname{arctg}\left(\operatorname{th} \frac{\pi\left(x-x_{0}\right)}{2 \alpha h} \operatorname{cotg} \frac{\pi z}{2 h}\right)-\operatorname{arctg}\left(\operatorname{th} \frac{\pi\left(x+x_{0}\right)}{2 \alpha h} \operatorname{cotg} \frac{\pi z}{2 h}\right)
$$

and

$$
\phi_{2}=\operatorname{arctg}\left(\operatorname{th} \frac{\pi\left(x+x_{1}\right)}{2 \alpha h} \operatorname{cotg} \frac{(z-h)}{2 h}\right)-\operatorname{arctg}\left(\operatorname{th} \frac{\pi\left(x-x_{1}\right)}{2 \alpha h} \operatorname{cotg} \frac{\pi(z-h)}{2 h}\right)
$$

are also solutions of equation (2). Solution $\phi_{1}$ describes the t-vector distribution in the whole sample created by the $(-\pi)$ and $(+\pi)$ twist disclination pair situated on the lower glass plate at $x=x_{0}$ and $x=-x_{0}$, respectively (see Sect. 2). Similarly $\phi_{2}$ gives the t-vector distribution of $(+\pi)$ and $(-\pi)$ twist disclination pair on the upper glass plate at $x=x_{1}$ and $x=-x_{1}$, respectively. The central and the outer parts of FLC in figure 3 a with mutually opposite polarisations are separated by twist walls. In this case the nucleus is formed by surface $\pi$-disclination quadrupole. By inserting (11) into the expression of the bulk elastic energy (expression (1) with $E=0$ ) we obtain the elastic energy of the nucleus per unit length in the $y$-direction

$$
F_{\mathrm{el}}=\pi\left(B_{1} B_{3}\right)^{1 / 2}\left\{-2 \ln \left|\frac{\operatorname{ch} \frac{\pi\left(x_{1}+x_{0}\right)}{2 \alpha h}}{\operatorname{ch} \frac{\pi\left(x_{1}-x_{0}\right)}{2 \alpha h}}\right|+\ln \left|\frac{\operatorname{sh} \frac{\pi x_{1}}{\alpha h} \operatorname{sh} \frac{\pi x_{0}}{\alpha h}}{\operatorname{sh}^{2} \frac{\pi r_{\mathrm{s}}}{2 \alpha h}}\right|\right\}
$$

In the special case when $x_{1}=x_{0}$ it is

$$
F_{\mathrm{el}}=2 \pi\left(B_{1} B_{3}\right)^{1 / 2} \ln \left|\frac{\operatorname{th} \frac{\pi x_{0}}{\alpha h}}{\operatorname{sh} \frac{\pi r_{\mathrm{s}}}{2 \alpha h}}\right| \text {. }
$$

If we assume the rectangular shape of the nucleus, as in section 2 , the elastic energy is in the form [15]

$$
F_{\mathrm{el}}=4 x_{0} \pi\left(B_{1} B_{3}\right)^{1 / 2} \ln \left|\frac{\operatorname{th} \frac{\pi x_{0}}{\alpha h}}{\operatorname{sh} \frac{\pi r_{\mathrm{s}}}{2 \alpha h}}\right|+4 x_{0} \pi B_{1} \ln \left|\frac{\operatorname{th} \frac{\pi x_{0}}{h}}{\operatorname{sh} \frac{\pi r_{\mathrm{s}}}{2 h}}\right|,
$$

where only the interaction between parallel disclinations has been taken into account.

The surface energy of the FLC on the upper glass is $4 R^{2} W_{1}+4 x_{0}^{2}\left(W_{2}-W_{1}\right)$ and that on the lower glass is $4 R^{2} W_{2}+4 x_{0}^{2}\left(W_{1}-W_{2}\right)$. The total surface energy $4 R^{2}\left(W_{1}+W_{2}\right)$ does not depend on the parameter $x_{0}$. The electric field energy can be simply estimated supposing that outside a nucleus it is $\phi=0$ and inside $\phi=\pi$. Then

$$
-\int P_{\mathrm{s}} E \cos \phi \mathrm{d} V=-P_{\mathrm{s}} E h\left(4 R^{2}-8 x_{0}^{2}\right) \text {. }
$$

The total energy $F=F_{\text {el }}-P_{\mathrm{s}} E h\left(4 R^{2}-8 x_{0}^{2}\right)+4 R^{2}\left(W_{1}+W_{2}\right)$ where $F_{\mathrm{el}}$ is given by equation (13). The orientation $\phi=\pi$ is favoured if $P_{\mathrm{s}} E<0$. Using the same procedure as in 
section 2 we obtain

$$
\left|E_{\mathrm{c}}^{\mathrm{II}}\right| \approx \frac{\pi\left(B_{1} B_{3}\right)^{1 / 2}}{4 r_{\mathrm{s}} h\left|P_{\mathrm{s}}\right|}\left(1+\frac{1}{\alpha}\right) \ln (2 e) .
$$

Expression (14) does not depend explicitly on the surface energy because of the nucleus special geometry (Fig. 3b).

\section{Nucleus in the sample bulk.}

A nucleation of this type has been proposed, namely in references [5-8, 11-13]. The structure of possible nuclei is schematically shown in figure 4 (note the shift of the coordinate origin as compared to Figs. 2 and 3). Two similar solutions of the equilibrium equation (2) can be proposed :

$$
\phi_{\mathrm{d}}=\operatorname{arctg}\left(\operatorname{th} \frac{\pi\left(x-x_{0}\right)}{\alpha h} \operatorname{cotg} \frac{\pi z}{h}\right)-\operatorname{arctg}\left(\operatorname{th} \frac{\pi\left(x+x_{0}\right)}{\alpha h} \operatorname{cotg} \frac{\pi z}{h}\right)
$$

and

$$
\phi_{\mathrm{w}}=\operatorname{arctg}\left(\operatorname{th} \frac{\pi\left(x-x_{0}\right)}{\alpha h} \operatorname{cotg} \frac{\pi|z|}{h}\right)-\operatorname{arctg}\left(\operatorname{th} \frac{\pi\left(x+x_{0}\right)}{\alpha h} \operatorname{cotg} \frac{\pi|z|}{h}\right) .
$$

The solution $\phi_{\mathrm{d}}$ describes the t-vector distribution around the $(+2 \pi)$ and $(-2 \pi)$-twist disclination pair situated at $x=-x_{0}$ and $x=x_{0}$, respectively (Fig. 4a). Disclinations are again parallel to the $y$-axis. At $z= \pm h / 2$ it is $\phi_{\mathrm{d}}(x, z= \pm h / 2)=0$. However, there is a discontinuity in $\phi_{\mathrm{d}}$ for $z \rightarrow 0$ which can be expressed by

$$
-\phi_{\mathrm{d}}\left(x, z \rightarrow 0_{+}\right)+\phi_{\mathrm{d}}(x, z \rightarrow 0)=-\pi\left[\operatorname{sg}\left(x-x_{0}\right)-\operatorname{sg}\left(x+x_{0}\right)\right] .
$$

The solution $\phi_{\mathrm{w}}$ can be obtained as the sum $\phi_{\mathrm{w}}^{+}+\phi_{\mathrm{w}}^{-}$with $\phi_{\mathrm{w}}^{ \pm}$given by

$$
\begin{aligned}
\phi_{\mathrm{w}}^{+} & =\operatorname{arctg}\left(\operatorname{th} \frac{\pi\left(x-x_{0}\right)}{\alpha h} \operatorname{cotg} \frac{\pi z}{h}\right)-\operatorname{arctg}\left(\operatorname{th} \frac{\pi\left(x+x_{0}\right)}{\alpha h} \operatorname{cotg} \frac{\pi z}{h}\right), z \geqslant 0, \\
& =0, \quad z<0
\end{aligned}
$$

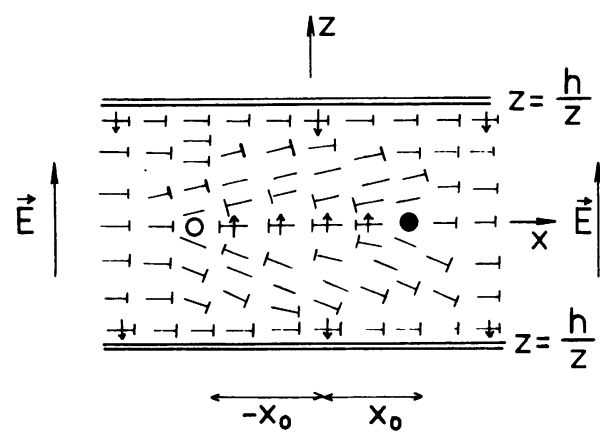

a)

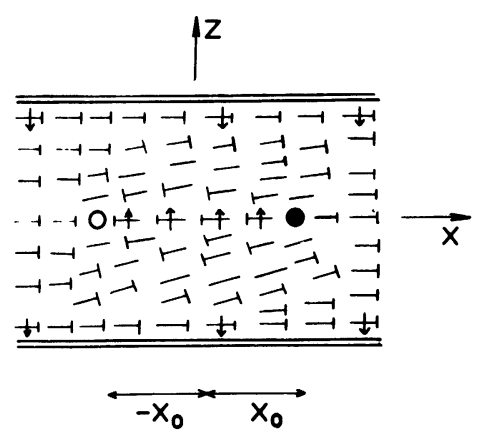

b)

Fig. 4. - Nucleation in the sample centre. a) Nucleus of the molecular structure with $\mathbf{P}_{\mathrm{s}}$ parallel to an applied electric field is bounded by a pair of $(+2 \pi)$ and $(-2 \pi)$-twist disclinations situated at $x=-x_{0}$ and $x=x_{0}$. b) The same nucleus as in a) is bounded by two singularities in $\phi$-distribution situated at $x=-x_{0}$ and $x=x_{0}$ on the plane $z=0$ where also the discontinuity in the torque $C_{x z}$ occurs. 
and

$$
\begin{array}{rlrl}
\phi_{\mathrm{w}}^{-} & =0, & & z>0, \\
& =-\phi_{\mathrm{w}}^{+}, \quad & z \leqslant 0 .
\end{array}
$$

The solutions $\phi_{\mathrm{w}}^{+}$and $\phi_{\mathrm{w}}^{-}$describe the $( \pm \pi)$-surface twist disclinations in samples with thickness $h / 2$. It can be found by comparing the solutions $\phi_{\mathrm{w}}^{ \pm}$with $\phi$ given by equation (3) in which $h$ is changed by $h / 2$. The sum $\phi_{\mathrm{w}}^{+}+\phi_{\mathrm{w}}^{-}$is then realized by putting the samples with thickness $h / 2$ together in order to obtain a sample of thickness $h$. The two singularities at $x=-x_{0}$ and $x=x_{0}$ correspond to $( \pm \pi)$ and $(\mp \pi)$ twist disclination pairs at these points. The nature of these singularities is demonstrated by the behaviour of $\phi_{\mathrm{w}}$ and $\partial \phi_{\mathrm{w}} / \partial z$ at $z \rightarrow 0$. For $z \rightarrow 0$ the function $\phi_{\mathrm{w}}$ takes the form

$$
\phi_{\mathrm{w}}(x, z=0)=\frac{\pi}{2}\left[\operatorname{sg}\left(x-x_{0}\right)-\operatorname{sg}\left(x+x_{0}\right)\right]
$$

with the discontinuity along the $x$-axis. The derivative

$$
\partial \phi_{\mathrm{w}} / \partial z=\frac{\partial \phi_{\mathrm{w}}}{\partial|z|} \operatorname{sg}(z)
$$

has a discontinuity at $z \rightarrow 0$ and it is

$$
\left(\frac{\partial \phi_{\mathrm{w}}}{\partial z}\right)_{z \rightarrow 0_{-}}-\left(\frac{\partial \phi_{\mathrm{w}}}{\partial z}\right)_{z \rightarrow 0_{+}}=(-2)\left(\frac{\partial \phi_{\mathrm{w}}}{\partial|z|}\right)_{z=0}
$$

where $\frac{\partial \phi_{\mathrm{w}}}{\partial|z|}$ is at $z=0$ :

$$
\left(\frac{\partial \phi_{\mathrm{w}}}{\partial|z|}\right)_{z=0}=\frac{\pi}{h}\left[-\operatorname{cth} \frac{\pi\left(x-x_{0}\right)}{\alpha h}+\operatorname{cth} \frac{\pi\left(x+x_{0}\right)}{\alpha h}\right] .
$$

At $z= \pm h / 2$ it is $\phi_{\mathrm{w}}(x, z= \pm h / 2)=0$.

The discontinuity (18) corresponds to the discontinuity in the torque $C_{z x}=B_{1} \frac{\partial \phi_{\mathrm{w}}}{\partial z}$ (introduced in Ref. [17]) on the plane $z=0$. If unbalanced, this torque leads to the instability of singularities. Thus the occurrence of this defect is induced by an application of an external torque realized in our case by an electric field.

Using the properties of both $\phi_{\mathrm{d}}$ and $\phi_{\mathrm{w}}$ the elastic energy of a nucleus in the sample centre per unit length in the $y$-direction can be evaluated :

$$
\begin{aligned}
F_{\mathrm{el}}=\int_{-h / 2}^{h / 2} \mathrm{~d} z \int_{-R}^{R} \mathrm{~d} x\left\{\frac{B_{1}}{2}\left(\frac{\partial \phi}{\partial z}\right)^{2}+\frac{B_{3}}{2}\left(\frac{\partial \phi}{\partial x}\right)^{2}\right\}= \\
=\frac{B_{1}}{2} \int_{-R}^{R} \mathrm{~d} x\left\{\left[\phi \frac{\partial \phi}{\partial z}\right]_{z=0_{+}}^{z=h / 2}+\left[\phi \frac{\partial \phi}{\partial z}\right]_{z=-h / 2}^{z=0}\right\} .
\end{aligned}
$$

The evaluation of the elastic energy (20) with the solution $\phi_{\mathrm{d}}$ can be carried out by using the discontinuity condition (16). Then the elastic energy (20) is in the form

$$
F_{\mathrm{el}}=\pi B_{1} \int_{-x_{0}}^{x_{0}}\left(\frac{\partial \phi_{\mathrm{d}}}{\partial z}\right)_{z=0} \mathrm{~d} x
$$


where $\left(\partial \phi_{d} / \partial z\right)_{z=0}$ has the same form as expression (19). The insertion of the solution $\phi_{\mathrm{w}}$ into the energy (20) gives

$$
\begin{aligned}
F_{\mathrm{el}}=\frac{B_{1}}{2} \int_{-R}^{R} \mathrm{~d} x\left\{\phi_{\mathrm{w}}(x, z=0)\left(\frac{\partial \phi_{\mathrm{w}}}{\partial z}\right)_{z \rightarrow 0_{-}}-\phi_{\mathrm{w}}(x, z=0)\left(\frac{\partial \phi_{\mathrm{w}}}{\partial z}\right)_{z \rightarrow 0_{+}}\right\} & = \\
& =B_{1} \int_{-x_{0}}^{x_{0}}\left(\frac{\partial \phi_{\mathrm{w}}}{\partial|z|}\right)_{z=0} \mathrm{~d} x,
\end{aligned}
$$

where expressions (17) and (18) were used. Then both solutions $\phi_{\mathrm{d}}$ and $\phi_{\mathrm{w}}$ give the same formula for $F_{\mathrm{el}}$ in the form :

$$
F_{\mathrm{el}}=2 \pi\left(B_{1} B_{3}\right)^{1 / 2} \ln \left|\frac{\operatorname{sh} \frac{2 \pi x_{0}}{\alpha h}}{\operatorname{sh} \frac{\pi r_{\mathrm{c}}}{\alpha h}}\right|
$$

where $r_{\mathrm{c}}$ is the $2 \pi$-twist disclination core radius.

As in sections 2 and 3 let us assume a rectangular nucleus. Its elastic energy is approximately

$$
F_{\mathrm{el}}=4 \pi x_{0}\left(B_{1} B_{3}\right)^{1 / 2}\left\{\ln \left|\frac{\operatorname{sh} \frac{2 \pi x_{0}}{\alpha h}}{\operatorname{sh} \frac{\pi r_{\mathrm{c}}}{\alpha h}}\right|+\frac{1}{\alpha} \ln \left|\frac{\operatorname{sh} \frac{2 \pi x_{0}}{h}}{\operatorname{sh} \frac{\pi r_{\mathrm{c}}}{h}}\right|\right\}
$$

The surface energy does not play any role in this case because $\phi_{\mathrm{d}}=\phi_{\mathrm{w}}=0$ at $z= \pm h / 2$. The energy of an electric field can be estimated by supposing a double twist in the sample volume over the nucleus area, i.e.

$$
\phi_{\mathrm{d}} \approx \frac{2 \pi}{h}\left(z-\frac{h}{2}\right) \text { or } \phi_{\mathrm{w}} \approx \frac{2 \pi}{h}\left(|z|-\frac{h}{2}\right) .
$$

Outside the nucleus it is $\phi=0$. Then

$$
-P_{\mathrm{s}} E \int \cos \phi \mathrm{d} V=4 h P_{\mathrm{s}} E\left(x_{0}^{2}-R^{2}\right)
$$

because

$$
\int_{-h / 2}^{h / 2} \cos \frac{2 \pi}{h}\left(z-\frac{h}{2}\right) \mathrm{d} z=0
$$

The extreme of the total energy $F=F_{\mathrm{el}}+4 h P_{\mathrm{s}} E\left(x_{0}^{2}-R^{2}\right)$ with respect to $x_{0}$ gives, as in the previous sections, the following estimation of the critical electric field :

$$
\left|E_{\mathrm{c}}^{\mathrm{III}}\right| \approx \frac{\pi\left(B_{1} B_{3}\right)^{1 / 2}}{2 r_{\mathrm{c}} h\left|P_{\mathrm{s}}\right|}\left(1+\frac{1}{\alpha}\right) \ln (2 e) .
$$




\section{Discussion.}

Using the same approximation and the same procedure of evaluation of the electric field interaction energy with FLC, we have obtained the maximum critical electric fields for three nucleation processes, i.e. the nucleation on a sample surface, over the whole sample thickness and in the sample bulk, which allows us to compare these nucleations.

In our calculations we have implicitly supposed a high anchoring energy barrier between the orientations $\phi=0$ and $\phi= \pm \pi$. This assumption leads in fact to nearly singular disclination cores of surface disclinations (Sects. 2 and 3) characterized by a core radius $r_{\mathrm{s}}$ which depends on the maximum anchoring energy $W_{\mathrm{M}}$ as $r_{\mathrm{s}} \approx\left(B_{1} B_{3}\right)^{1 / 2} / 2 W_{\mathrm{M}}$ (Refs. [17, 18]). Thus the critical field $E_{\mathrm{c}}$ given by expressions (10) and (14) depends linearly on $W_{\mathrm{M}}$ as $\left|E_{\mathrm{c}}\right| \sim W_{\mathrm{M}} / h\left|P_{\mathrm{s}}\right|$. However, in other models the quadratic dependence of $E_{\mathrm{c}}$ on $W_{\mathrm{M}}$ was found, i.e. $E_{\mathrm{c}}=8 W_{\mathrm{M}}^{2} / 27 B_{1} P_{\mathrm{s}}$. In the models of $[19,20]$ the deformation of the molecular structure under the influence of an external electric field is calculated exactly but only in the one-dimensional approach. In these models the switching from one uniform structure to the other is achieved when FLC molecules overcome the surface anchoring energy barrier $W_{\mathrm{M}}$ under the influence of an external electric field.

In our approach, after a nucleus is created, the further motion of the defects surrounding the structure of the opposite polarity will be easy because high torques near the disclination core help the molecules to overcome the anchoring energy barrier between both uniform states. If $W_{\mathrm{M}}$ is small, $r_{\mathrm{s}}$ will be higher.

By comparing the approximate values of the upper limit of the critical fields given by expressions (10), (14) and (22) a behaviour of the thin FLC sample under an external electric field can be estimated. A disadvantage of our two-dimensional approach in comparison with $[19,20]$ is the assumption that the FLC structure is not deformed by an electric field after the nucleus is formed and the approximate estimate of the interaction energy of an electric field with FLC. On the other hand, our approach permits comparison of different disclination nucleation mechanisms by using the value of a critical field. In this note we have investigated three nucleation processes characterized by critical fields $E_{\mathrm{c}}^{\mathrm{I}}, E_{\mathrm{c}}^{\mathrm{II}}$ and $E_{\mathrm{c}}^{\mathrm{III}}$ given by expressions (10), (14) and (22), respectively. It is seen that

$$
\left|E_{\mathrm{c}}^{\mathrm{I}}\right|<\left|E_{\mathrm{c}}^{\mathrm{II}}\right| \text {. }
$$

If an electric field reaches the values $E_{\mathrm{c}}^{\mathrm{I}}$ given by equation (10) the nucleation of the structure with orientation $\phi=-\pi$ starts at the lower surface where the molecular anchoring with higher energy $W_{2}$ occurs. Molecules switch to $W_{1}$ - anchoring energy minimum on this surface. The same conclusion was found in references [3, 22]. If $E$ does not further increase, the twisted state is induced in large domains of FLC sample. Further increase of the electric field leads to the molecular switching also on the other glass surface and the switching of the whole sample to the orientation $\phi=-\pi$ is achieved (Fig. 3).

The relation between $\left|E_{\mathrm{c}}^{\mathrm{II}}\right|$ and $\left|E_{\mathrm{c}}^{\mathrm{III}}\right|$ determines which of these two mechanisms occurs. Expressions (14) and (22) differ namely in values $r_{\mathrm{s}}$ and $r_{\mathrm{c}}$, respectively. While the core radius $r_{\mathrm{s}}$ of $\pi$-surface twist disclination can be associated with the surface energy, the core radius $r_{\mathrm{c}}$ of $2 \pi$-twist disclination in the sample bulk is the parameter of the model developed in section 3 .

When $r_{\mathrm{c}}$ is of the order of $r_{\mathrm{s}}$ then $\left|E_{\mathrm{c}}^{\mathrm{II}}\right|<\left|E_{\mathrm{c}}^{\mathrm{III}}\right|$ and for a slow increase of $E$ the switching proceeds only through the surface nucleation. Such a situation was observed in references [ 3 , 4]. On the other hand, when e.g. $r_{\mathrm{c}} \geq 2 r_{\mathrm{s}}$ then $\left|E_{\mathrm{c}}^{\mathrm{III}}\right| \leqslant\left|E_{\mathrm{c}}^{\mathrm{II}}\right|$. Then for gradually increased $E$ the transition to the twisted state is followed by the $2 \pi$-twist disclination nucleation in the 
sample bulk what leads to the FLC structure in the sample with both surface and bulk disclinations as observed for instance in references [6-8].

When $E$ is decreased to $E=0$, the orientation $\phi=-\pi$ remains. Further decrease of $E$ leads at $-E_{\mathrm{c}}^{\mathrm{I}}$ to the nucleation of the structure $\phi=0$ on the upper surface of the sample and we can discuss nucleations similarly as described above.

When applying a sharp pulse of high value of an electric field very quickly, as high as the maximum of $\left|E_{\mathrm{c}}^{\mathrm{II}}\right|$ and $\left|E_{\mathrm{c}}^{\mathrm{III}}\right|$, all types of nucleation described in sections 2-4 can be realized simultaneously. Therefore in the case of a sharp pulse of high electric field the switching process in FLC sample seen in an optical microscope can be very complex. Detailed optical observations [6-8] and also the numerical simulation of switching process [22] confirm such a FLC behaviour. However, further spreading of the domain with the orientation $\phi=-\pi$ in the sample depends on the disclination mobility. If the $2 \pi$-disclination mobility is greater then the surface $\pi$-disclination mobility, the nucleus in the sample bulk will have a dominant influence on further dynamics of switching.

It should be noted, however, that our models based on simplifications $(i-v)$ cannot describe the whole complexity of textures observed in [6-13] namely because the problems connected with «zigzag » are not treated here. Namely the «zigzag » defect contains $2 \pi$ disclinations [11-13] which can support the other $2 \pi$-disclination nucleation in the sample bulk during switching. For this reason the critical field $\left|E_{\mathrm{c}}\right|$ of switching with the presence of « zigzag " defect in the sample can be smaller when compared with $\left|E_{\mathrm{c}}^{\mathrm{III}}\right|$ obtained in our model.

Up to now we have discussed disclination nucleation processes in thin FLC samples covered by glasses with anchoring properties which do not change under an external electric field. Such a surface anchoring energy can be approximated, analogously to $[15,18]$, as

$$
W_{\mathrm{s}}=W_{\mathrm{M}} \sin ^{2} \phi \mp W_{0} \cos \phi,
$$

where $W_{M}$ and $W_{0}$ are positive. The sign (-) is valid for the upper cover glass and (+) for the lower glass. Then it is seen from (23) that $W_{1}=-W_{0}$ and $W_{2}=W_{0}$. However, we can imagine such a surface treatment producing a FLC anchoring energy dependent on the external electric field. Such a treatment can be realized e.g. by depositing a thin layer of transparent solid state ferroelectrics on cover glasses. In this case expression (23) should be modified by adding an additional term e.g. $-\left|P_{\mathrm{s}}\right| P \cos \phi$, where $P$ is the spontaneous polarisation of the solid ferroelectrics. Then

$$
W=W_{M} \sin ^{2} \phi \mp W_{0} \cos \phi-\left|P_{s}\right| P \cos \phi .
$$

Once oriented along an external electric field on both cover surfaces the polarisation $\mathbf{P}$ helps to orientate $\mathbf{P}_{\mathrm{s}}$ of FLC in the same direction. When the electric field is switched off, $\mathbf{P}$ remains nonzero and stabilizes the uniform structure with $\mathbf{P}_{\mathrm{s}}$ parallel to $\mathbf{P}$ satisfying the condition $\left|P_{\mathrm{s}} P\right|>W_{0}$ (Fig. 5a).

When the field of opposite polarity is applied, the reversal of both $\mathbf{P}$ and $\mathbf{P}_{\mathrm{s}}$ takes place and after switching off the external field $\mathbf{E}$, a uniform FLC structure with the opposite polarisation is stabilized (Fig. 5b). In our approximation the change of the anchoring energy during switching influences only the nucleation described in section 2. This effect is an example of the « surface bistability " hinted at by Dahl [21]. A uniform FLC structure stabilized by such surfaces with the anchoring changed by an external electric field can be called an actively surface stabilized ferroelectric liquid crystal (ASSFLC) structure. This ASSFLC structure can produce an interesting memory effect if such a « surface bistability » can be realized. 


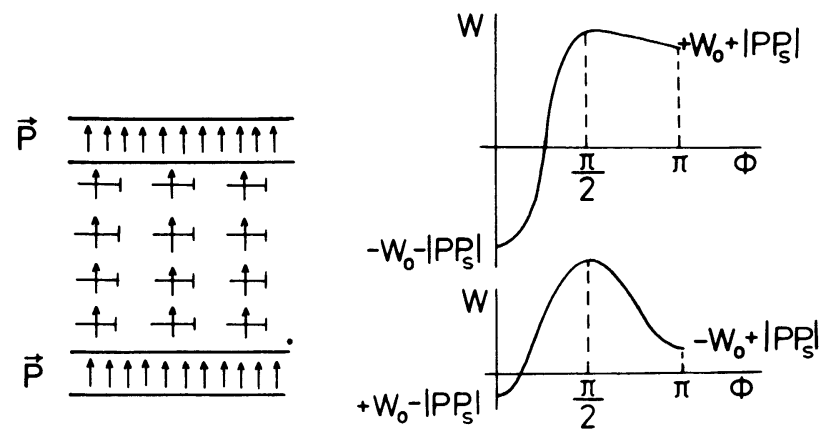

a)

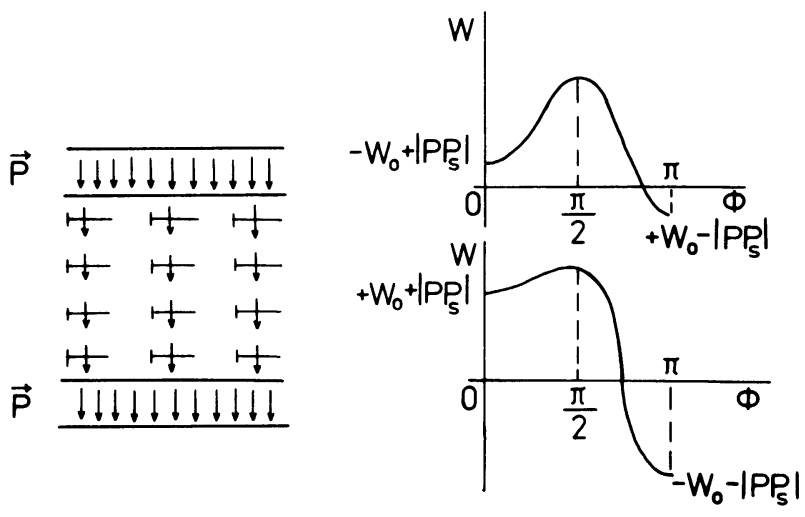

b)

Fig. 5. - Uniform FLC structure stabilized by the interaction with solid state ferroelectrics and described by the orientation. a) $\phi=0$, b) $\phi=\pi$ (left) and corresponding schematic courses of the anchoring energies for the upper and lower surfaces (right) both having absolute minima at a) $\phi=0, \mathrm{~b}) \phi=\pi$.

\section{References}

[1] Handschy, M. A. and Clark, N. A., Ferroelectrics 59 (1984) 69.

[2] Clark, N. A. and Lagerwall, S. T., Ferroelectrics 59 (1984) 25.

[3] Glogarová, M. and Pavel, J., J. Phys. France 45 (1984) 143.

[4] Glogarová, M., Fousek, J., LejČek, L. and Pavel, J., Ferroelectrics 58 (1984) 161.

[5] Ouchi, Y., Ishikawa, K., TAKezoe, H., Fukuda, A., Kondo, K., ERA, S. and MukoH, A., Jpn J. Appl. Phys. 24 (1985) Suppl. 24-2, 899.

[6] Takezoe, H., Ouchi, Y., Ishikawa, K. and Fukuda, A., Mol. Cryst. Liq. Cryst. 139 (1986) 27.

[7] Ouchi, Y., Takano, H., Takezoe, H. and Fukuda, A., Jpn J. Appl. Phys. 26 (1987) L21.

[8] Ouchi, Y., Takezoe, H. and Fukuda, A., Jpn J. Appl. Phys. 26 (1987) 1.

[9] Rieker, T. P., Clark, N. A., Smith, G. S., Parmar, D. S., Sirota, E. B. and Safinya, C. R., Phys. Rev. Lett. 59 (1987) 2658.

[10] Clark, N. A. and Rieker, T. P., Phys. Rev. A 37 (1988) 1053.

[11] Ouchi, Y., Takezoe, H. and Fukuda, A., Ferroelectrics 85 (1988) 113.

[12] Hiji, N., Chandani, A. D. L., Nishiyama, S.-I., Ouchi, Y., Takezoe, H. and Fukuda, A., Ferroelectrics 85 (1988) 99. 
[13] OuCHI, Y., PhD Thesis, Tokyo (1987).

[14] LEJCEK, L., Ferroelectrics 58 (1984) 139.

[15] Fousek, J. and LeJČEK, L., Proc. 6th IEEE Int. Symp. on Applications of Ferroelectrics, Bethlehem (1986).

[16] Pavel, J., J. Phys. France 45 (1984) 137.

[17] KlÉman, M., Points, Lines, and Walls in Liquid Crystals, Magnetic Systems and Various Ordered Media (J. Wiley and Sons, New York) 1983.

[18] LejCek, L., Glogarová, M. and Pavel, J., Phys. Status Solidi (a) 82 (1984) 47.

[19] SChiller, P., Cryst. Res. Technol. 21 (1986) 167.

[20] Galvan, J. M., Thèse, Talence (1986).

[21] DAHL, I., comment in Proc. 1th Int. Symp. on FLC, Arcachon (1987).

[22] Yamada, Y., Tsuge, T., Yamamoto, N., Yamawaki, M., Orihara, H. and Ishibashi, Y., Jpn. J. Appl. Phys. 26 (1987) 1811. 